\section{Uterovesicovaginal fistula following placenta percreta: case review and management options}

\author{
Sonika Mann, ${ }^{1}$ Pushpa Dahiya, ${ }^{1}$ \\ Amit Mann, ${ }^{2}$ Shikha Madan' \\ 'Department of Obstetrics and \\ Gynaecology, PGIMS Rohtak; \\ 2Department of Nephrology, PGIMS \\ Rohtak, Haryana, India
}

\begin{abstract}
We report case of uterovesicovaginal fistula following caesarean section with review of management options. The case presented with continuous dribbling of urine with cyclical menouria immediately following dilatation and curettage for retained placental bits. Cystoscopy along with hysteroscopy and methylene blue instillation through the cervix confirmed the diagnosis. Patient was put on prolonged bladder drainage for three months. The fistulas healed spontaneously requiring no further surgical management. All over the world prevalence of the utero-vesical fistula is increasing with frequent use of caesarean section.
\end{abstract}

\section{Introduction}

A urinary fistula is an abnormal communication between any part of urinary system and skin or some internal hollow viscus. They are uretero-vaginal, vesico-vaginal or urethravaginal. Utero-vesical fistulas are the least common type of urogenital fistulas, comprising less than $4 \%$ of all urogenital fistulas. ${ }^{1}$ They are most commonly caused by repeated caesarian sections, which are increasing in modern day obstetrics. ${ }^{2}$ The other obstetrical causes include protracted or neglected labor and lower segment caesarian section (LSCS). Placenta percreta with bladder invasion can also lead to vesico-uterine fistula. ${ }^{3}$ Other causes include endometriosis, contraceptive devices, malignant tumors, rupture of the uterus and bladder after obstructed labor, dilatation and curettage, placenta percreta invading bladder, radiation therapy and iatrogenic trauma of intermittent bladder self cathterisation. ${ }^{4}$

Ureterovaginal fistula without the leakage of urine, cyclical hematuria and amenorrhea is a rare condition referred to as Youssef's syndrome, the most common cause being LSCS. The gynecological causes are associated with the pelvic surgery e.g. total hysterectomy, anterior colporrhaphy etc., radiotherapy for treat- ment of carcinoma cervix, direct neoplastic infiltration of carcinoma of cervix, dilatation and curettage, contraceptive devices and iatrogenic trauma of intermittent bladder self cathterisation. ${ }^{4}$ The treatment of VUF is generally surgical closure of fistula tract with interposition of graft, hysterectomy if family is complete. ${ }^{2}$ Conservative management has also been mentioned. ${ }^{3}$

\section{Case Report}

A 29-year-old patient presented to our hospital with history of continuous dribbling of urine and cyclical menouria for 2 months. She had undergone 2 caesarian sections, the last one being 2 months back for major degree of placenta praevia. One week post operatively patient developed on and off bleeding per vaginum and check curettage was done by some practitioner for the same. Dribbling of urine started immediately following the curettage and menouria occurred 3 weeks later. On examination patient had a well-healed vertical scar on the abdomen. On vaginal examination there was continuous dribbling of urine from the vagina, no definite fistulous tract was identified. Uterus was normal size, anteverted with restricted mobility. Ultrasonography (USG) showed a deficiency in the posterior wall of bladder. Intravenous pyelography showed a fistulous tract between the posterior wall of bladder and vagina. On cystoscopy a defect was seen near the posterior wall of bladder near the ureteric orifice. Methylene blue dye injected into the bladder was seen leaking through the upper third of vagina and no dye was elicited leaking into the uterus. Hysteroscopy revealed a defect in the anterior wall of the uterus at the level of isthmus measuring about $1 \mathrm{~cm}$. Methylene blue was then pushed into the uterus through cervix and it leaked into the bladder confirming the fistulous tract. The final diagnosis of two fistulas, vesicovaginal fistula and utero-vesical fistula both was made. As patient was only 3 months postpartum, decision for conservative management was made so that inflammation and edema of the tissues could settle down. The bladder was catheterized with self-retaining Foley's catheter. The patient was given oral contraceptive pills for contraception. The catheter was omitted after 3 months following bladder training. During the training patient was asymptomatic and hence repeat hysteroscopy and cystoscopy was not performed. The patient was symptom free after removal of the catheter and had normal voiding function. She was called for monthly follow up for next three months when she was found to have normal voiding function and hence not subjected to repeat cystocopy and hysteroscopy.
Correspondence: Sonika Mann, Department Obstetrics and Gynaecology, PGIMS Rohtak, 138/22 Vikas Nagar Rohtak, Haryana 124001, India.

Tel. +91.09896004974.

E-mail: drsonikamann@gmail.com

Key words: vesicovaginal fistula, placeta percreta, obstetrics fistula.

Received for publication: 7 June 2013.

Revision received: not required.

Accepted for publication: 8 August 3013.

This work is licensed under a Creative Commons Attribution NonCommercial 3.0 License (CC BYNC 3.0).

CC Copyright S. Mann et al., 2013

Licensee PAGEPress, Italy

Urogynaecologia 2013; 27:e2

doi:10.4081/uij.2013.e2

\section{Discussion}

Three types of the fistula are distinguished: type I) with menouria; type II) with dual flow via both the bladder and vagina; and type III) with normal vaginal menses. ${ }^{5}$ Symptoms depend upon the level of fistula and can be explained by the sphincteric mechanism of the uterine isthmus ${ }^{6}$ and the different pressure gradients. The menstrual blood accumulates in the uterine cavity and when the pressure raises above $25-30 \mathrm{~mm} \mathrm{Hg}$, the sphincter of the isthmus relaxes and a bloody discharge occurs. When a fistula is present above the level of isthmus, the menstrual blood passes directly from the uterine cavity into the bladder. The result is Youssef's syndrome that is amenorrhea with patent cervical canal, menouria and the absence of urinary leakage through the vagina. $^{7}$

When the fistula is located below the level of isthmus, the menstrual blood accumulates normally in the uterine cavity, and when the sphincter relaxes, the menstrual blood passes as it should through the cervix into the vagina and not through the fistula into the bladder. Conversely when submitted to high pressure in the bladder, urine leaks from the bladder into the uterine cervix and vagina presenting mainly as dribbling of urine.

In some cases menouria may be without amenorrhea, can be associated with urinary leak through the vagina as was the case in our patient. The symptoms can appear early after surgery or months and years later.

Diagnosis can be achieved by cystoscopy, vaginoscopy, hysteroscopy, cystography, and excretory urography. However findings from any of these procedures can be inconclusive. Transvaginal sonography may reveal an abnor- 
mal hypoechoeic line connecting the endometrial cavity to the bladder, appearing vascularised on Doppler study. Magnetic resonance imaging (MRI) may show focal hypotense area in the uterus with the interruption of the uterine wall. However USG and MRI findings may also be difficult to differentiate from the normal post caesarian findings. Injection of contrast medium in the uterus or the bladder is necessary to show the fistula and to visualize the fistulous tract. Whether to opacify the bladder or the uterus depends on the symptoms. In case of menouria, hysterography reveals the diagnosis but to visualize the fistulous tract helical computed tomography with sagital reformation is helpful. ${ }^{4}$ When fistula is complicated with vaginal leak of urine, opacification of the bladder is the clue to the diagnosis. Excretory urography can be falsely normal but is required to rule out ureteric fistula.

Management includes conservative treatment as well as surgical repair. Spontaneous healing is reported in 5\% of cases. ${ }^{8}$ Conser vative management by bladder catheterization for at least 4 to 8 weeks is indicated when the fistula is discovered just after delivery since there is a good chance of spontaneous closure of the fistulous tract. Hormonal treatment with continuous oral contraceptives for 6 months should be tried in Youssef's syndrome to induce a period of amenorrhea that allows closure of fistula. ${ }^{8}$

Surgery is the mainstay and standard treatment for these patients. ${ }^{9}$ Patients scheduled for surgery should undergo pretreatment of any urinary infection. Molina ${ }^{10}$ reported successful treatment of vesico-uterine fistula following caesarian after cystoscopic identification and fulguration if the tract. Tanaka et al. ${ }^{11}$ reported disappearance of incontinence after cystoscopic fulguration of the fistula but it resulted in temporary curve and subsequent surgical repair was needed. $\mathrm{Ra}^{9}$ presented retrospective analysis of 12 cases of post caesarian vesico-uterine fistule.

Surgical repair of vesicouterine and vesicovaginal fistulas are performed by different approaches, which include the vaginal, retropubic extraperitoneal transvesical, and transperitoneal. Transperitoneal access is considered the most effective with vesico- uterine fistulas. ${ }^{8}$ The use of flaps is recommended in all but smallest fistulae. In vaginal route
Martius graft $^{12}$ or gracilis muscle ${ }^{13}$ is used. Omental transposition between bladder and uterus has produced excellent results. The omentum with its good blood supply has a natural role in resolving abdominal infection. Lent ${ }^{14}$ presented a case of successful closure by transvesical approach without interposition of omental graft. El-lateef ${ }^{15}$ used retropubic extra peritoneal transvesical approach using anterior abdominal wall fat graft. Recently, numerous reports of laparoscopic and robot-assisted surgical repair of these fistulas have shown that these techniques can be used with efficacy and safety with added advantages of short hospital stay, reduced morbidity associated with surgical incision and lower blood loss. ${ }^{8}$ Hysterectomy is curative in Youssef's syndrome if there is no requirement of future pregnancy. Ozmen16 presented such case, which was treated with hysterectomy and repair of bladder defect. The pregnancy rate after repair is $31.25 \%$ with a rate of term deliveries of $25 \%$.

\section{Conclusions}

Vesicouterine fistulae are no longer a rare diagnosis and occur most commonly secondary to lower segment caesarean section. The standard treatment for delayed presentation fistula is surgical repair of the fistulae, whereas conservative approach can be advocated for fistulas presenting immediate postpartum. Prevention is better than cure meticulous practice of obstetric and surgical principles during caesarean section can prevent the formation of these fistulae. If damage to the bladder is suspected, every effort should be made to confirm this, as immediate repair of the bladder at this stage is easy and almost invariably successful.

\section{References}

1. Dodero D, Corticelli A, Caproale E, et al. Vesico-uterine fistula and bladder endometriosid. Minerva Ginecol 2001;53:137-40.

2. Syed Majeed SMK, Subhani SS. An unusal case of Youssef's syndrome (vesicouterine fistula) and its relationship with placenta percreta. JCPSP 2007;17:168-9.

3. Youssef AF. Menouria following lower segment caesarean section: a syndrome. Am J Obstet Gynecol 1957;73:759-67.

4. Smayra T, Ghossain MA, Buy JN, et al. Vesicouterine fistulas: Imaging findings in three cases. AJR 2005;184:139-42.

5. Józwik M, Józwik M. Clinical classification of vesicouterine fistula. Int $\mathrm{J}$ Gynaecol Obstet 2000;70:353-7.

6. Youssef AF. The uterine isthmus and its sphincter mechanism: a radiographic study. The uterine isthmus under normal conditions. Am J Obstet Gynecol 1958;75:1305-19.

7. Machado LM. Vesicouterine fistulas after caesarean section. Rev Gynaecol Obstet 1935;29:471-73.

8. Dorairajan LN, Hemal AK. Lower urinary tract fistula: the minimally invasive approach. Curr Opin Urol 2009;19:556-62

9. Rao M, Prasad MS et al. Post caesarean vesicouterine fistule-Youssef syndrome: our experience and review of published work. ANZ J Surg 2006;76:243-5.

10. Molina LR, Lynne CM, Politano VA. Treatment of vesicouterine fistula by fulguration. J Urol 1989;141:1422-23.

11. Tanaka K, Chokyu H, Yoshimura K, et al. Vesicouterine fistula a case report. Hinyokiki Kiyo 1996;2:601-03.

12. Zimmerin PE, Ganabathi K, Leach GE. Vesicovaginal fistula repair. Urol Clin North Am 1994;2:87-9.

13. Leach GE, Trockman BA. Surgery for vesicovaginal and urethrovaginal fistule and urethral diverticulum. In: Walsh PC, Retik AB, Vaughan ED, Wein AJ, eds. Campbell's urology, 7th ed. Philadelphia: WB Saunders Co.; 1998. pp 1135-53.

14. Lent V, Laaser M. Uterovesical fistula. Aktuelle Urol 2005;36:quiz 65-6.

15. El-Lateef Moharram AA, Abd El-raouf MA. Retropubic repair of genitourinary fistula using a free supporting graft. BJU Int 2004;93:581-3.

16. Ozmen E, Yalcinkaya F, Gulhan N, Unal S. Vesicouterine fistula (Youssef's syndrome) case report. Int Urol Nephrol 1998;30:451-53. 